\title{
Dense hadron star in quark degree of freedom
}

\author{
Yiharn Tzeng ${ }^{1, a}$ and S.Y.Tsay Tzeng ${ }^{2}$ \\ ${ }^{1}$ Institute of Physics, Academia Sinica, Taipei 11529, Taiwan, ROC \\ ${ }^{2}$ Dept. of Electro-Optical Engineering, National Taipei University of Technology, Taipei 10608, Taiwan, ROC
}

\begin{abstract}
The quark degree of freedom may play an important role as one studies dense hadron stars which can help to understand the universe origin. We add a temperature dependence to the effective quark mass adopted from a quark-quark interaction on the QCD basis to probe properties of the star in the quark degree of freedom. Based on this interaction, the quark matter's equation of state is obtained and its thermodynamic characteristics is investigated in detail. Stability of a star made of such matter is examined with and without strange quarks. The Tolman-Oppenheimer-Volkov equation along with the condition that $d m / d r=4 \pi r^{2} E$ are used to calculate mass and radius of such a star. Exact computations are made to calculate the star's radius and mass at several temperatures. Comparisons of results from these temperatures are made and the significance is carefully investigated and discussed.
\end{abstract}

\section{Introduction}

Studies on neutron stars or hadron stars are usually working in three lines, namely, in the quark's, the hadron's, or the mixed degrees of freedom. At the center of the star, the particle density could be much higher than the ordinary nuclear matter so that the quark effects may be prominent. We thus choose to study the star in the quark degree of freedom. Because information from experimental observations have been quite limited, quite large percentage of related studies were concentrated on zero temperature as in [1]. These stars could be temporarily equilibrium at some finite temperatures before cooling down. Studying these stars at finite temperatures can enhance our understanding of their evolutions. A density dependent strange mass was used in [2] and finite temperature properties of the stars were examined by approximating the integration in series. Here we modify the effective quark mass used in [1] to have temperature dependence and perform exact computations. Except $u, d$ quarks and electrons, we also test the stability of the star with and without strange quarks. Results are as what expected.

\section{Theories and Computations}

The grand potential density for the system is

$$
\Omega=-\sum_{i} \frac{g_{i} T}{2 \pi^{2}} \int_{0}^{\infty} \ln \left[1+\exp \left(-\beta\left(e_{i}-\mu_{i}\right)\right)\right] p^{2} d p,
$$

\footnotetext{
ae-mail: tzengyh@phys.sinica.edu.tw
} 
with $e_{i}=\sqrt{m_{i}^{2}+p^{2}}, g_{i}, m_{i}, \mu_{i}$ being the energy, the degeneracy, the effective mass, and the chemical potential of quark $i, \beta=\frac{1}{T}, T$ the temperature.

The average particle number density of quark $i$ is given as

$$
n_{i}=-\frac{\partial \Omega}{\partial \mu_{i}}=\frac{g_{i}}{2 \pi^{2}} \int_{0}^{\infty} \frac{e^{-\beta\left(e_{i}-\mu_{i}\right)}}{1+e^{-\beta\left(e_{i}-\mu_{i}\right)}} p^{2} d p .
$$

Particles reach equilibrium via $d+s \leftrightarrow u+e+\bar{v}_{e}, s+u \leftrightarrow u+d$. Thus the chemical potentials are $\mu_{d}=\mu_{s}=\mu_{u}+\mu_{e} \equiv \mu$. Particle number densities are subject to constraints from equilibrium and charge neutrality. We thus have

$$
\frac{1}{3}\left(n_{u}+n_{d}+n_{s}\right)=n_{b}, \quad \frac{2}{3} \mu_{u}-\frac{1}{3} \mu_{s}-\frac{1}{3} \mu_{d}-\mu_{e}=0,
$$

with the baryon number density $n_{b}=N_{b} / V, N_{b}$ the total baryon number, and $V$ the volume of the system.

The energy density of the system is

$$
\varepsilon=\Omega+\sum_{i} n_{i} \mu_{i}-T \frac{\partial \Omega}{\partial T}
$$

or

$$
\varepsilon=\frac{\partial \beta \Omega}{\partial \beta}+\sum_{i} n_{i} \mu_{i}
$$

with

$$
\frac{\partial \beta \Omega}{\partial \beta}=\sum_{i} \frac{g_{i}}{2 \pi^{2}} \int_{0}^{\infty} \frac{\left(e_{i}-\mu_{i}\right) e^{-\beta\left(e_{i}-\mu_{i}\right)}}{1+e^{-\beta\left(e_{i}-\mu_{i}\right)}} p^{2} d p .
$$

The pressure $P=<\frac{\partial E}{\partial V}>$ with $E, V$ the total energy and the volume of the system, can be obtained from $P=-\frac{\partial \Omega V}{\partial V}$.

If $\Omega$ and $m_{i}$ are functions of $n_{b}$, the pressure $P$ further becomes

$$
P=-\Omega+n_{b} \frac{\partial \Omega}{\partial n_{b}}=\sum_{i} \frac{\partial \Omega}{\partial m_{i}} \frac{\partial m_{i}}{\partial n_{b}}=\sum_{i} \frac{g_{i}}{2 \pi^{2}} \frac{\partial m_{i}}{\partial n_{b}} \int_{0}^{\infty} \frac{\frac{m_{i}}{e_{i}} e^{-\beta\left(e_{i}-\mu_{i}\right)}}{1+e^{-\beta\left(e_{i}-\mu_{i}\right)}} p^{2} d p .
$$

The zero temperature effective quark mass was parameterized in [1] as $m_{q}=m_{q 0}+\frac{D}{n_{b}^{1 / 3}}$, with $m_{q 0}$ being quark $q$ 's current mass, and the extra term being deduced from the hamiltonian of the quark's interaction part. As the interaction may vary with temperature, We add in a temperature dependence $F(T)$ to it and rewrite it as

$$
m_{q}=m_{q 0}+\frac{D}{n_{b}^{1 / 3}} F(T)
$$

With this effective quark mass, the quark can be viewed as equivalent to a free particle with the energy $e_{i}=\sqrt{m_{i}^{2}+p^{2}}$, and thus the above thermodynamics quantities can be calculated.

The quark current masses used in our calculations are $m_{u}=5 \mathrm{MeV}, m_{d}=10 \mathrm{MeV}$, and $m_{s}=$ $50 \mathrm{MeV}$. The mass of electron is set to be $m_{e}=0.5 \mathrm{MeV}$. To meet the limitation on quark's effective mass (such as in [1]) we set $F(T)=1-\frac{T}{T_{c}} \exp \left(-\frac{T}{T_{c}}\right)$, with $T_{c}$ being the temperature that the phase transition between hadrons and quark-gluon plasma starts. 
At a fixed $T$, for a given baryon particle density, one can find corresponding chemical potentials $\mu$ and $\mu_{e}$ from (3). The energy density and the pressure can then be obtained from (5) and (7).

Using satisfactory results from above computations, one then solve $\varepsilon(r)$ and $P(r)$ from the TolmanOppenheimer-Volkoff (TOV) equation

$$
\frac{d P(r)}{d r}=-\frac{G m(r) \varepsilon(r)\left[1+\frac{P(r)}{\varepsilon(r)}\right]\left[1+\frac{4 \pi r^{3} P(r)}{m(r)}\right]}{r^{2}\left[1-\frac{2 G m(r)}{r}\right]},
$$

together with

$$
\frac{d m}{d r}=4 \pi r^{2} \varepsilon(r)
$$

Finally from

$$
M=m(R)=4 \pi \int_{0}^{R} \varepsilon(r) r^{2} d r
$$

one has the mass of the star as at $r=R$, the pressure $P=0$.

\section{Results and discussions}

Our results are plotting in figs. 1 to 2 . We start the central baryon density $n_{b}$ a little bit above that of the normal nuclear matter, and raise it up to about 1.3 per $\mathrm{fm}^{3}$. Shown on the left of fig. 1 is the star radius as a function of the starting baryon density at the center of the star for three different temperatures, $T=2 \mathrm{MeV}, 20 \mathrm{MeV}$, and $40 \mathrm{MeV}$. The radius is under $15 \mathrm{~km}$ for the temperature up to $20 \mathrm{MeV}$, and the higher $T$, the lager radius. We also compare the radius when s quarks are included in the quark matter to that when no s quarks are present, for examples, curves (a) to (b) and (d) to (e). Apparently, it is smaller as s quarks are included.

Shown on the right of fig. 1 is the star mass versus $n_{b}$ for several different $T$ 's. Similar to those seen the radius part, the higher $T$, the heavier the star mass. Qualitatively, particles under higher temperature $T$ are less stable, and thus have larger energy densities $(\varepsilon)$ and higher pressure $(P)$. To reach $P=0$ hence needs longer distance. The star composed of such particles therefore has heavier mass and larger radius. Including s quarks also results in a less heavy star, as curves (a) to (b), and (d) to (e). The star mass is less than 1.8 solar mass $M_{\text {sun }}$ at $T=2 \mathrm{MeV}$ when including s quarks, and becomes larger for higher $T$.

The star mass versus its radius is plotted in fig. 2. Properties appearing in fig. 1, namely, the higher $T$, the larger radius and mass, as well as including s quarks reducing the radius and the mass, all preserve here.

The common features apparently seen in all of the above figures from our calculations are: the star being more stabilized as the temperature being lowered, and as s quarks being Included. These are consistent with what one expects.

The temperature dependence function $F(T)$ appearing in (8) seems to work reasonably in our considered temperature range. However, it needs more information from astronomical observations and further theoretical efforts to improve and extend it to a wider temperature range. We are currently working on this line.

\section{References}

[1] G.X.Peng, H.C.Chiang, J.J. Yang, L. Li, and B. Liu, Phys. Rev. C61, 015201 (1999). G.X.Peng, H.C.Chiang, B.S.Zou, P.Z.Ning, and S.J.Luo, ibid, C62, 025801 (2000).

[2] G.Lugonees and O.G. Benvenuto, Phys. Rev. D52, 1276 (1995). 

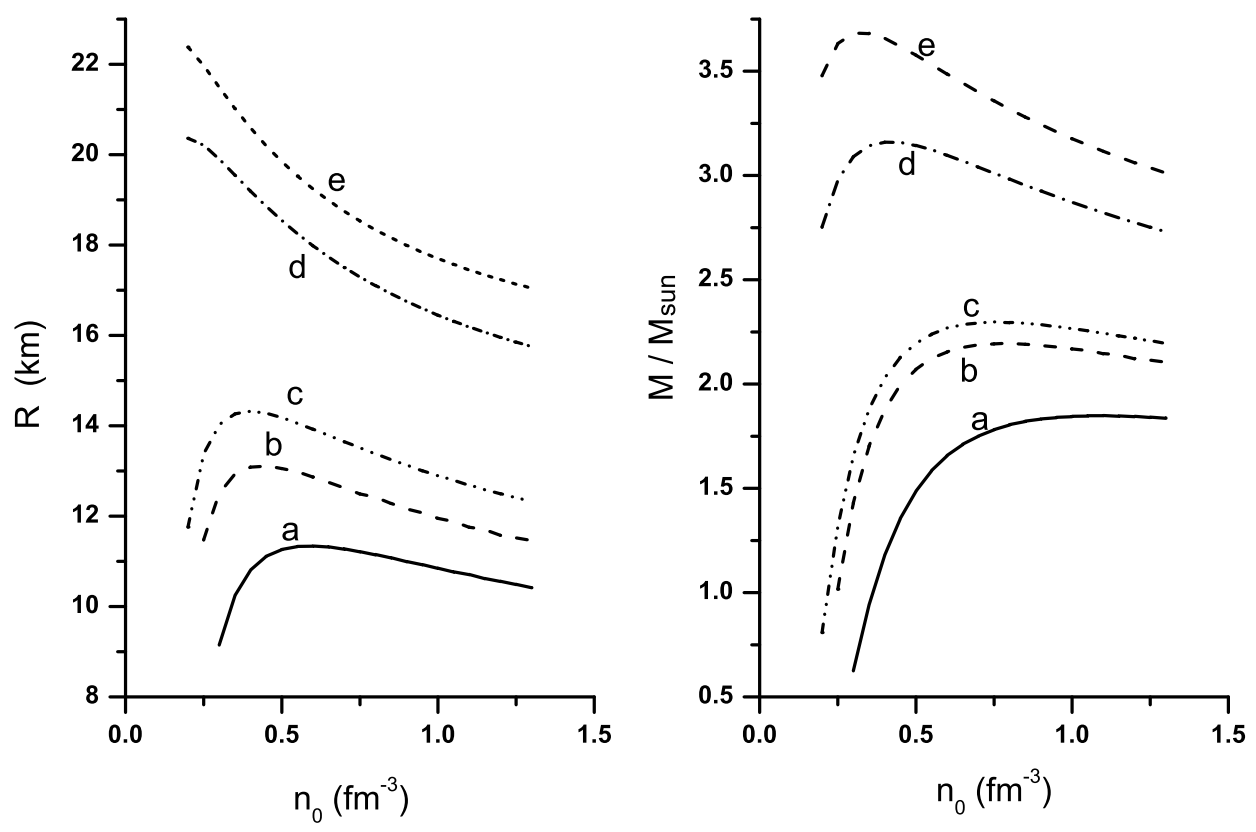

Figure 1. Radius(left) and mass(right) of the star as functions of the baryon density at the star's center under different temperatures. (a)T $=2 \mathrm{MeV}$, (b) same as (a), but without s quarks, (c) $\mathrm{T}=20 \mathrm{MeV}$, (d)T=40 MeV, (e) same as (d), but without s quarks.

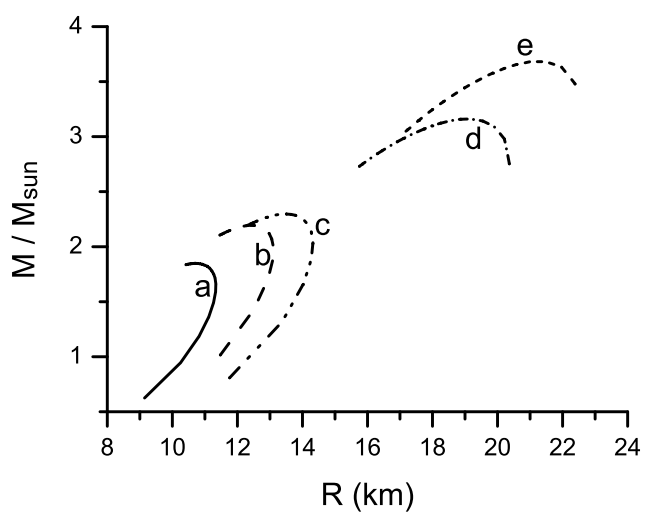

Figure 2. Relationship between the ratios of the star's to the sun's masses and the star's radius under the same conditions as those in Fig. 1 